\title{
¿NUEVAS FORMAS DE ORGANIZACIÓN, VIEJAS CONSECUENCIAS PARA EL TRABAJADOR?
}

\author{
MARÍA ALICIA DÍAZ \\ MARIANA SABAS \\ NADIA NEIFF
}

FACULTAD DE CIENCIAS ECONÓMICAS UNIVERSIDAD NACIONAL DEL NORDESTE

ARGENTINA

"La confianza entre las personas se sustenta a partir del cumplimiento de las promesas, del soporte de la integridad individual y del nivel de responsabilidad con el que se asumen los vínculos”.

Verónica y Florencia Andrés. 


\section{INTRODUCCIÓN}

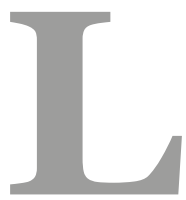

os Call Centers (CC) constituyen un sector en claro desarrollo, con una fuerte expansión mediante la tercerización (Korinfeld, Alimena y Marcos, 2001). Según Del Bono (2005), el desarrollo de los Call Centers, se enmarca en la tendencia de aumento de la creación de empleos en el sector servicios y se vincula directamente con los procesos de reorganización empresarial, que se han visto posibilitados con la adopción generalizada de nuevas tecnologías de la información y de la comunicación (Tic). De esta manera, se puede señalar que "dos fuerzas convergentes impulsaron y delinearon las características más importantes del origen y crecimiento de los Call Center: la reestructuración de las grandes firmas y los procesos de reorganización del trabajo, que dicha reestructuración conlleva" (Del Bono, 2005, p. 03).

La instalación de este servicio externalizado en nuestro país se produjo a principios del año 2001-2002, en un contexto de crisis económica y social y devaluación monetaria. Este contexto propició la llegada de inversores extranjeros, que encontraron en nuestro país un lugar con posibilidades económicas muy favorables para instalar sus áreas de servicios. En este sentido, nuestro país ofrecía a estas empresas costos laborales competitivos, recursos humanos altamente calificados, moderna infraestructura de telecomunicaciones, beneficios impositivos.

En la región del NEA la ciudad de Resistencia, a partir del año 2008, se convirtió en pionera al brindar el servicio de Call Center externalizado generando oportunidades de empleo a miles de jóvenes de la región (Oviedo, 2009).

La forma peculiar que adoptan estas organizaciones, define el trabajo prescripto, en su perfil y contenido, y a partir de éste se derivan las exigencias, requerimientos y limitaciones propias del puesto, que configuran la carga global de trabajo de un teleoperador. Específicamente, este trabajo describe las repercusiones de la carga mental y psíquica de trabajo de los teleoperadores, por la importancia que adquieren estas dimensiones en la ejecución de la tarea.

Este trabajo de investigación se realizó en el marco de una tesina de grado y tuvo como objetivo general describir las repercusiones de la carga mental y psíquica en teleoperadores de Call Centers externalizados de la ciudad de Resistencia (Chaco) en relación a la forma de organización del trabajo. En consecuencia, nos guiaron los siguientes objetivos específicos: a) identificar los aspectos más significativos de la organización del trabajo; b) distinguir y describir los indicadores más relevantes de la carga mental y psíquica del puesto de teleoperadores; y c) describir de qué manera repercuten estas dos dimensiones de la carga de trabajo en los teleoperadores. 


\section{ORGANIZACIÓN Y CONTENIDO DE TRABAJO}

La forma de organización del trabajo que adopta una empresa u organismo, determina una serie de características en relación a la tarea que realiza el trabajador, que terminan por configurar la carga global de trabajo. En esta línea, lo que se pretende indagar y profundizar en esta investigación es particularmente la organización a nivel tarea, es decir, a nivel del puesto de trabajo; teniendo en cuenta esta interacción e interrelación que existe entre los tres niveles superiores (global, departamental, tarea) (Chiavenato, 1995).

A nivel global de organización se presentan a grandes rasgos dos modelos disímiles con diferentes características y estructura organizacional: una forma de organización tradicionalrígida y las nuevas formas de organización flexible (Chiavenato, 1995, 2006). El primer modelo está caracterizado por los principios básicos planteados por Taylor y Ford y el segundo modelo está orientado a lo que se presume que son las "nuevas forma de organizar el trabajo". El modelo de organización que una empresa u organismo adopte, va a afectar al modo de organizar las tareas que se realizan en ella. Por lo que, en esta investigación no se pretende hacer un análisis exhaustivo de los modelos de estructura organizacional que se desarrollan en los Call Centers externalizados, pero no se puede dejar de lado por la interrelación planteada.

Para hacer el análisis a nivel de tarea o puesto de trabajo, se utiliza el concepto empleado por Neffa de "organización y contenido de trabajo". En cuanto a la organización, según este autor, existen dos elementos que permiten caracterizarla al nivel específico del puesto de trabajo: división social y división técnica del trabajo.

En relación al contenido del trabajo, Neffa (1988) menciona una serie de elementos relevantes que lo incluyen. Estos factores son: La naturaleza de la tarea prescripta; las calificaciones profesionales requeridas por el puesto de trabajo para que se lleve a cabo la tarea prescripta; las mayores o menores posibilidades que tienen los trabajadores que ocupan el puesto, para utilizar y desarrollar en el mismo las calificaciones profesionales que disponen; el grado de responsabilidad que se asigna al trabajador en virtud del puesto de trabajo que ocupa; el carácter individual o colectivo del puesto de trabajo, así como las comunicaciones y cooperación que deben establecer con los demás trabajadores; el grado de autonomía, de interdependencia o de subordinación del trabajador y las posibilidades normales de promoción profesional que son inherentes al puesto.

Como se expuso anteriormente, la forma de organización del trabajo que adopta una empresa u organismo, determina una serie de características en relación a la tarea que realiza el trabajador, que terminan por configurar la carga global de trabajo. 


\section{CARGA GLOBAL DE TRABAJO}

Siguiendo a Neffa (1988) la Carga Global de Trabajo hace referencia a: "el conjunto de repercusiones de las exigencias, requerimientos y limitaciones del puesto de trabajo, determinadas por el trabajo prescripto” (p.93). Así, la carga global de trabajo es el resultado de la "acción articulada de todos los factores de riesgo del medio ambiente de trabajo y de los factores de las condiciones de trabajo que están presentes en una empresa u organización y repercuten sobre el colectivo de trabajo y cada uno de los trabajadores”(p.93).

El autor diferencia tres dimensiones de la carga de trabajo: física, psíquica y mental. En este trabajo, se analizará sólo la carga mental y la carga psíquica de trabajo de los teleoperadores tercerizados, ya que para determinar la carga física es necesario recurrir, complementariamente, a otros profesionales especialistas como ser médicos del trabajo, psicólogos y ergónomos.

\section{CARGA MENTAL Y PSÍQUICA}

Siguiendo a Neffa (1988), Carga Mental, la carga mental tiene relación con las estructuras mentales y los procesos cognitivos, éstos pueden ser divididos en etapas: percepción de la información, identificación y reconocimiento de las informaciones percibidas, adopción de decisiones, la actividad o realización de la tarea.

A continuación se citan brevemente los indicadores de la carga mental y psíquica, presentados por Neffa (1988):

Carga Mental-Indicadores: apremio o las exigencias de tiempo; complejidad-rapidez de la tarea; atención; minuciosidad y memoria operatoria.

Mientras que la carga del trabajo mental depende fundamentalmente de los requerimientos cognitivos derivados de la organización de las tareas para quienes ocupan los puestos de trabajo, la Carga Psíquica, tiene relación con los aspectos psico-sociales de la carga de trabajo, que se relacionan muy estrechamente con el contenido de trabajo (Neffa, 1988).

Siguiendo a Neffa (1988), los principales factores determinantes y condicionantes de la Carga Psíquica de Trabajo son: grado de iniciativa; grado de ambigüedad acerca de los resultados de la tarea realizad; status social o el reconocimiento social de la actividad; comunicación y cooperación; relaciones o contacto con los usuarios, clientes y el público en general; contradicción emergente de la situación provocada por las necesidades (natural o artificialmente generadas) insatisfechas y responsabilidad en cuanto al manejo de dinero.

Las empresas modifican y adaptan las formas de organización del trabajo a las exigencias y dinamismo de la economía y de la sociedad actual. Precisamente, los Call Centers surgen en un contexto de globalización económica y como producto de los procesos de reorganización 
empresarial resultante de la utilización de las Tic y el desarrollo de políticas de tercerización y deslocalización.

\section{CALL CENTER -TELEOPERADOR}

Según lo define Del Bono y Bulloni (2008):

"Los Call Centers son un soporte tecnológico desarrollado por las empresas para satisfacer determinados aspectos de la relación con sus clientes en forma no presencial; son plataformas telefónicas que pueden ser gestionadas directamente por la empresa interesada en brindar atención a sus clientes, o por empresas proveedoras, en las que se ha tercerizado su gestión” (p. 03).

Dentro de la estructura de los Call Centers, la figura del teleoperador cobra importancia. En general, los teleoperadores son jóvenes de entre 18 a 25 años o personas mayores desocupadas. En su mayoría, los teleoperadores son mujeres, quienes buscan la posibilidad de complementar el salario familiar, estudiantes que buscan un ingreso con el que puedan lograr independizarse o que les permita seguir estudiando con un trabajo part-time (medio tiempo). Mayoritariamente, quienes trabajan en los Call Centers son estudiantes universitarios o recién recibidos (Del Bono y Bulloni, 2008; Del Bono, 2005).

En síntesis, se puede decir que los cambios producidos en la economía, los avances tecnológicos y los procesos de reorganización empresarial, fueron delineando diferentes formas de organizar el trabajo. En este sentido, los teleoperadores representan una nueva figura laboral, que adquiere relevancia, en esta nueva sociedad de la información y la comunicación bajo una situación de estricto control, a partir de la automatización del trabajo, el manejo continuo de información, el contacto constante con los clientes, satisfaciendo sus necesidades con formas estructuradas de comunicación, margen prácticamente nulo de decisión, etc. Todas estas características de las tareas del teleoperador, están determinadas por el trabajo prescrito e inciden directamente en la carga mental y psíquica de los mismos.

\section{METODOLOGÍA}

Se realizó un estudio Cuali-cuantitativo - Descriptivo. La utilización de una metodología mixta permitió la complementación de los métodos cualitativo y cuantitativo. Por un lado, el método cualitativo, a través de entrevistas en profundidad, permitió hacer un análisis com- 
plejo y holístico de la problemática planteada. Por otro lado, con el método cuantitativo, mediante encuestas auto-administradas, se obtuvo un panorama general sobre la forma de organizar el trabajo de los teleoperadores de Call Center externalizados de la ciudad de Resistencia.

Por lo tanto, las fuentes de datos primarios fueron las entrevistas en profundidad y las encuestas auto-administradas efectuadas a los teleoperadores. También se realizaron dos entrevistas a personal del área de Recursos Humanos de estas organizaciones. A partir de ellas se pudo obtener información sobre las particularidades de la organización y del puesto. Como fuentes secundarias se utilizaron diarios de la región, revistas especializadas en el sector servicios, datos extraídos del sitio oficial de Dirección de Prensa y Difusión del Gobierno la Provincia del Chaco.

Fueron nuestra unidad de análisis los teleoperadores de Call Centers externalizados de la ciudad de Resistencia. La muestra fue seleccionada de las cuatro empresas más importantes localizadas en la ciudad: Atento (con aproximadamente 450 empleados), Allus Global (330 empleados), Apex América (con 300 empleados), y Call Connect -más conocida como Kalvo(120 empleados).

Para ser parte de la muestra, los teleoperadores debían reunir los siguientes requisitos (criterios de muestreo): desempeñarse actualmente en alguna de las empresas citadas, (era importante que la persona se desempeñe como teleoperador al momento de realizarse el trabajo de campo, para determinar la actual repercusión de la carga mental y psíquica como así también detectar los elementos de la organización de trabajo), con una antigüedad mayor a los 3 meses (superando el periodo de prueba) y tener entre 18 y 35 años (que según antecedentes de investigaciones es el rango de edad más frecuente en estas organizaciones).

La selección de la muestra, para los datos cuantitativos, se realizó al Azar Simple (Sabino, 1996). Se obtuvieron un total de 88 encuestas, examinadas a través del análisis estadístico. Para los datos cualitativos se utilizó un muestreo tipo "bola de nieve". Se realizaron un total de once entrevistas en profundidad a teleoperadores de los distintos Call Center externalizados (seleccionando los casos hasta obtener la saturación teórica), analizando posteriormente los datos obtenidos con la aplicación de la técnica de teorización anclada (Mendicoa, 2003; Pujadas Muñoz, 1992).

Los aspectos estudiados fueron: la organización del trabajo y los indicadores de carga mental y carga psíquica que se evidenciaron como relevantes en el estudio de campo.

\section{RESULTADOS Y CONCLUSIONES}

A continuación se exponen los resultados obtenidos, para lo cual resulta necesario aclarar que a partir de abarcar las particularidades de los puestos, se hará referencia a la figura del teleoperador, cuando no hacemos distinciones por puestos. En los casos en que se requiere hacer una distinción por puestos, éstos se presentarán como: teleoperador de atención al cliente 
de primera o segunda línea y telemarketer (teleoperador de ventas).

Del análisis realizado sobre la organización del trabajo, se destaca una marcada división social y técnica del trabajo. En cuanto a la división social, los teleoperadores no participan en la planeación de las tareas, sólo se encargan de ejecutar el trabajo prescripto, bajo estricta supervisión. El disgregar las funciones de diseño, planificación y ejecución, da origen a un conflicto entre el trabajo prescripto y el trabajo realmente ejecutado, ya que el trabajo prescripto, de ningún modo puede ser ejecutado tal como fue planificado, puesto que en la ejecución del mismo surgen contratiempos y situaciones fortuitas que requieren que el teleoperador aplique sus conocimientos, experiencias y creatividad, para el logro del objetivo planteado.

En relación a la división técnica, la parcelización de las tareas es intensiva. Los teleoperadores tienen asignadas tareas delimitadas y precisas, formando parte de una porción mínima del proceso de trabajo de teleatención, que se circunscribe a la realización de tareas rutinarias relacionadas con la atención al cliente o la venta de productos.

En función a esta forma de organizar el trabajo (con una marcada división técnica y social), la naturaleza de la tarea del teleoperador se torna monótona, repetitiva y sin margen de decisión. Respondiendo a elementos esenciales del modo de organización taylorista. A través de su aplicación, las empresas de Call Center logran la supresión del margen de autonomía e iniciativa de los teleoperadores, obteniendo la estandarización y mecanización del ritmo, de la calidad y del nivel de productividad del trabajo, alcanzando una mayor eficiencia gracias al crecimiento de la habilidad de cada trabajador.

En un contexto de competitividad global, centrado en la innovación tecnológica y de productos, el medio demanda estructuras flexibles que sean capaces de adaptarse a los cambios del entorno. Es por ello que para perdurar, los Call Center deben contar con un alto grado de adaptabilidad y flexibilidad, sin disminuir el nivel de productividad y calidad. En este sentido, los Call Center estratégicamente seleccionan a jóvenes de entre 18 y 25 años con estudios universitarios, que les garantizan un nivel de productividad mayor y un servicio de alta calidad, por los conocimientos, habilidades y destrezas adquiridos en la universidad. Sin embargo, esto genera un desajuste entre el nivel de formación superior que poseen y el requerido por el puesto, por lo que los teleoperadores se encuentran sobrecalificados. Asimismo, adoptan contratos con duración determinada, como por ejemplo: eventuales, por temporada o a plazo fijo, obteniendo mayor movilidad y flexibilidad laboral a bajo costo; generando inestabilidad laboral y competencia entre compañeros en el logro de los objetivos.

A partir de lo analizado, se puede decir que la adopción de algunos aspectos de una nueva forma organización, como lo es contar con personal altamente calificado y modalidades de contratación flexible, sólo constituyen una "fachada". Por un lado, poseen un diseño organizacional con características flexibles, para responder eficazmente a las demandas del mercado, pero la organización del trabajo a nivel tarea, entraña las características más básicas y elementales de las viejas formas de organizar el trabajo, repercutiendo en el teleoperador a nivel psíquico y mental. 
En los Call Centers externalizados predomina la forma de organización tayloristafordista, con una acentuada división técnica y social del trabajo, que en conjunto con el uso generalizado e intensivo de las Tics, da como resultado una elevada exigencia de ritmo de trabajo. A través del sistema ACD (Automatic Call Distributor), imponen un continuo y constante flujo de llamadas, que el teleoperador no puede controlar ni interrumpir. Asimismo, el cumplimiento del "tiempo ideal" de duración por llamada, también es una exigencia de cadencia impuesta. Mediante software especialmente diseñado, los supervisores realizan un control estricto de estos parámetros de tiempo, como así también de todas las actividades que realizan los teleoperadores, ya que no pueden tomar descansos, interrumpir una llamada en curso, abandonar o ausentarse momentáneamente de su puesto de trabajo sin la autorización de su superior. De esta manera, los teleoperadores se encuentran bajo un proceso de trabajo totalmente automatizado, con grandes exigencias y apremios de tiempo, siendo éste uno de los indicadores más relevantes, que indudablemente intensifican la carga mental.

Los teleoperadores de atención al cliente de primera línea poseen mayores exigencias de tiempo, ya que el objetivo del puesto es atender la mayor cantidad de llamadas en cortos plazos de tiempo. En cambio, para los teleoperadores de segunda línea, la exigencia se concentra en la calidad de llamada, quedando en segundo plano la extensión de la misma. Para los telemarketer, el condicionante externo de apremio y exigencia de tiempo es el sistema de remuneración, ya que deben cumplir una determina cantidad de ventas mensuales en forma grupal y/o individual, destacándose éstas últimas, que conforman las comisiones e influyen sensiblemente en su salario. Estos condicionamientos externos, generan un ambiente competitivo, que promueve el individualismo, en detrimento de la formación de un equipo de trabajo, donde el desempeño individual es un elemento clave para alcanzar un mayor salario. De esta manera se puede apreciar que la recompensa por el logro de los objetivos se ubica bajo la vieja concepción del hombre considerando sólo la motivación económica, y dejando de lado el reconocimiento social por parte de su supervisor y compañeros.

Para la ejecución de la tarea el teleoperador requiere constantemente hacer uso de su memoria operatoria para recordar datos, procedimientos, frases y operatorias, asimismo debe interactuar continuamente con sistemas informáticos y el cliente, sin distracciones o interrupciones. Esta interacción cliente-teleoperador-software, requiere que el teleoperador permanentemente detecte, procese, decodifique e intérprete una gran cantidad de información, para tomar decisiones rápidas, según sea el objetivo del puesto: satisfacer la necesidad del cliente o efectivizar una venta, cumpliendo las métricas de cantidad y calidad establecidas. Se puede decir, que los teleoperadores poseen una tarea con una elevada complejidad, por el gran volumen de información que manejan, hacen uso intenso de la memoria operatoria, requiriendo una gran atención y concentración para responder de manera rápida y efectiva, dentro de los límites impuestos de tiempo.

Por lo antes expuesto, se puede concluir que el teleoperador se encuentra bajo múltiples exigencias y requerimientos del puesto, generando un alto grado de carga mental. 
Como consecuencia de la forma de organizar y el contenido del trabajo, los teleoperadores deben realizar su tarea de manera individual, sin interrupciones, siguiendo operatorias e instrucciones que el sistema les va indicando; de tal manera que no tienen la posibilidad de comunicarse o contar con la cooperación de sus pares, por lo que recae sobre él toda la responsabilidad del cumplimiento del objetivo del puesto y los parámetros de calidad, cantidad, con un ritmo de trabajo impuesto. Esta condición en la forma de organizar el trabajo, no contribuye a generar un colectivo de trabajo, limitando la comunicación y cooperación, elevando la carga psíquica del teleoperador.

Asimismo, se debe destacar que existe una ambigüedad en los objetivos del puesto. La definición de dichos objetivos está sujeta a cambios que se presentan continuamente, como parte de la adecuación del servicio de cada campaña; también los supervisores o leaders tiene diferentes criterios de exigencias, lo que produce un desconcierto en el teleoperador que debe adaptarse constantemente a estos criterios.

Otro indicador relevante en la carga psíquica es la escasa autonomía que posee el puesto. Como se dijo anteriormente, los sistemas informáticos determinan el ritmo de trabajo, pautas, procedimientos y las frases que el teleoperador debe cumplir y seguir. Por esta condición impuesta, no pueden modificarlas, tomar decisiones o realizar procedimientos alternativos, sólo deben ceñir su accionar a las opciones del sistema. En este sentido, los teleoperadores deben "alinearse" a las opciones de un sistema, lo que lleva a sentirse como "máquinas" repetidoras.

Dentro de la estructura de un Call Center, los teleoperadores corresponden a la base operativa, y a través del desarrollo de su trabajo cumplen la función principal del proceso de teleatención, es decir que el puesto del teleoperador resulta de vital importancia. Sin embargo, existe una subvaloración de la tarea y de las calificaciones del teleoperador, por parte la sociedad. De esta manera, el bajo status social que tienen es un indicador de gran importancia en la carga psíquica.

Además de ser un puesto de suma relevancia para la estructura del Call Center, los teleoperadores son el contacto que los clientes tienen con las empresas que le brindan u ofrecen un servicio, su tarea es de constante interacción. En este sentido, los teleoperadores son quienes se encuentran expuestos a recibir consultas, quejas y reclamos, e inclusive agravios, de los clientes hacia las empresas prestatarias u oferentes del servicio. Para sobrellevar ésta situación los teleoperadores, deben desarrollar una serie de habilidades personales, inclusive se ven obligados a dar respuestas inapropiadas hasta mentir. Bajo múltiples presiones y exigencias, la atención se vuelve despersonalizada, siguiendo procedimientos totalmente prescriptos y cumpliendo estrictos parámetros de tiempos y calidad. Estar expuesto a estas situaciones, genera un aumento de la carga psíquica.

En función a lo analizado, se puede decir que el teleoperador asume un elevado grado de carga psíquica.

Los inconvenientes que se producen en el sistema por fallas técnicas, la gran cantidad de llamadas atendidas y realizadas durante toda la jornada en continuo contacto con los clientes, los constantes mecanismos cognitivos (selección de frases y procedimientos, procesamiento y 
carga de datos, memoria operatoria, interpretación del cliente), la exigencia de responder de manera efectiva en cortos plazos de tiempo, bajo un control estricto, sin poder interrumpir la labor, comunicarse o tomar decisiones de manera autónoma, no serían significativos si se tomaran en forma aislada. Sin embargo, teniendo en cuanta la Visión Renovadora de las CyMAT, estas condiciones no pueden estudiarse de manera aislada e individual. Es necesario partir de una concepción global e integrada, reconociendo la generación de los efectos sinérgicos entre los factores cuando se combinan. En este sentido, las condiciones de trabajo que se presentan bajo el modelo de organización peculiar que tienen los Call Center, toman significación en la carga mental y psíquica, cuando se los considera de manera conjunta y global, teniendo en cuenta su interrelación dinámica y simultánea, que multiplica los efectos específicos.

Se puede considerar, a través del análisis de lo estudiado, que no se visualiza una real nueva forma de organizar el trabajo sino simples adaptaciones y una convergencia o mutación de ambos modelos, ante las exigencias que el medio globalizado impone. Adaptando su estructura para perdurar en el mercado, mientras que la organización del trabajo sigue siendo tayloristafordista. La adopción de una forma de organizar el trabajo híbrida, que adopta características de una organización flexible y las combina con aspectos de una forma de organización rígida, pretende maximizar las características de cada una para obtener mayores y mejores resultados en cuanto a la productividad, eficiencia y eficacia en la consecución de sus objetivos.

Los Call Center externalizados de Resistencia, se caracterizan por hacer una medición cuantitativa de los resultados y objetivos, enfocándose en el cumplimiento del trabajo prescripto, que no refleja el esfuerzo mental y psíquico del teleoperador para el desarrollo de su tarea.

En este sentido se puede concluir que el teleoperador, asume una elevada carga global de trabajo; con exigencias, requerimientos y limitaciones impuestas por el trabajo prescripto, destacándose de manera significativa la carga mental y psíquica; con elevados apremios y exigencias de tiempo, tarea con un alto nivel complejidad, necesidad de responder con rapidez, la atención y memoria operatoria que debe aplicar en el desarrollo de la tarea, como así también el constante contacto con los clientes, la escasa comunicación y cooperación, bajo estatus social, nulo grado de autonomía y deficiente de comunicación de objetivos y logros.

\section{REFERENCIAS BIBLIOGRÁFICAS}

Chiavenato, I. (1995). “Administración de Recursos Humanos” Edición nº 4. México, DF.: McGraw-Hill.

Chiavenato, I. (2006). “Administración de Recursos Humanos” Edición nº 7. México, DF.: McGraw-Hill. 
Del Bono, A. (2005, Agosto). "Innovaciones tecnológicas y organizacionales Impacto sobre el trabajo y el empleo de jóvenes y mujeres en el sector servicios: La industria de los Call centers en Argentina". [Versión electrónica] Ponencia presentada en el $7^{\circ}$ Congreso Nacional de Estudios de Trabajo. Buenos Aires, Argentina. Recuperado de www.aset.org.ar/congresos/7/16005.pdf

Del Bono, A. y Bulloni, M. (2008). "Experiencias laborales juveniles Los agentes telefóni-cos de Call Centers offshore en Argentina”. [Versión electrónica] Trabajo y Sociedad: indagación sobre el trabajo, la cultura y las prácticas políticas en sociedades segmentadas. $\mathrm{N}^{0} 10$, vol. IX, Santiago del Estero, Argentina ISNN 1514-6871 (Caicyt). Recuperado de www.unse. edu.ar/trabajoysociedad/DEL_BONO.pdf

Del Bono, A. y Henry, L. (2008). "Tercerización de los servicios en la Argentina: empleo y gestión de los RRHH en los Call Centers" [Versión electrónica] Proyecto de investigación sobre: "Sistema de información para la evaluación y el monitoreo del empleo, el trabajo y la inclusión social", del Ministerio de Trabajo, Empleo y Seguridad Social. CONICET-CEILPIETTE-UNLP. Buenos Aires, Argentina. ). Recuperado de http://www.trabajo.gov.ar/left/ estadisticas/descargas/toe/toe_09_04.pdf

Korinfeld S.; Alimena, S; \& Marcos, M. F. (2001). "El nuevo paradigma económico-productivo y las condiciones de trabajo”. Ponencia presentada en $5^{\circ}$ Congreso Nacional de Estudios de Trabajo A.S.E.T. Buenos Aires, Argentina. Recuperado de www.aset.org.ar/congresos/5/aset/PDF/ALIMENA.PDF

Mendicoa, G. E. (2003) "Sobre tesis y tesistas: lecciones de enseñanza-aprendizaje". Buenos Aires: Espacio Editorial.

Neffa, J. C. (1988) “¿Qué son las condiciones y medio ambiente de trabajo?” Buenas Aires, Argentina: Humanitas.

Oviedo, L. A. (2009, Agosto). "El proceso de socialización laboral de los teleoperadores de Call y Contact Centers de las ciudades de Corrientes y Resistencia”. [Versión electrónica] Ponencia presentada en el $9^{\circ}$ Congreso de ASET. Buenos Aires, Argentina. Recuperado de www.aset.org.ar/congresos/9/Ponencias/p10_Oviedo.pdf

Pujadas Muñoz, J.J (1992). Cuadernos metodológicos. "El método biográfico: El uso de las historias de vida en ciencias sociales”. Madrid: CIS, Centro de Investigaciones Sociológicas.

Sabino, C. (1996). “Cómo hacer una tesis y elaborar todo tipo de escritos”. 4ta. Edición. Argentina, Buenos Aires: Lumen-Humanitas. 


\section{CURRICULUM VITAE}

\section{María Alicia Díaz}

Lic. en Relaciones Laborales (UNNE, 2014). Adscripta Cátedras Condiciones y Medio Ambiente de Trabajo (2010-2014). Carrera Relaciones Laborales, UNNE.

ma.aliciadiaz@gmail.com

\section{Mariana E. Sabas}

Lic. en Relaciones Industriales (UNNE, 1997). Profesora Adjunta Cátedras Condiciones y Medio Ambiente de Trabajo y Seminario de Práctica Profesional, Carrera Relaciones Laborales, UNNE. marianaesabas@yahoo.com.ar

\section{Nadia Neiff}

Licenciada en Relaciones Laborales (UNNE, 2010). Auxiliar Docente de Primera Categoría Cátedra Condiciones y Medio Ambiente de Trabajo, Carrera Relaciones Laborales, UNNE. nneiff@eco.unne.edu.ar 